\title{
A Paper On "Design and Implementation of Automatic Security Alert System Using GSM Network"
}

\author{
Abhilasha Walke ${ }^{1}$,Prof. Aruna D. Tete ${ }^{2}$, Vinod Takarkhede ${ }^{3}$ \\ ${ }^{1}$ PG Scholar, Department of Electronics Engineering, G. H. Raisoni College of Engineering, Nagpur, India \\ 440016 \\ ${ }^{2}$ Asst. Professor Department of Electronics Engineering, G. H. Raisoni College of Engineering, Nagpur, India \\ 440016 \\ ${ }^{3}$ Sr. Software Architect(Consultant),
}

\begin{abstract}
Real-time surveillance is an important aspect of an intelligent building with modern security demands. This paper presents the design and Implementation of Automatic Security Alert System Using PCI Board, GSM Network and Cross Platform Technology. To solve the problem of complex cabling, misdeclaration and missing alarm of traditional alarm system, GSM network is used. The project deals with a complete end-toend detection and alerting application which employs advanced technologies. Telecommunication card is used for the proper communication between the two ends as it offers communication between a numbers of places with the control centre. Cross platform is used as it is convenient to use single source code on multiple operating systems. In the following paper the application for fire is considered, where architecture for an Intellectual Voice Response System (IVRS) is being proposed. This system has been successfully installed in one of the corporation which is very useful for the society.

Index Terms: Fire alert system, GSM module, smoke detector, dialogic card, Cross platform.
\end{abstract}

\section{Introduction}

Nowadays, in the area of digital electronics and intelligent systems, building automation has become one of the fastest developing application-based technologies in the world. Automated security surveillance systems play an important role in providing an extra layer of security to the existing surveillance systems. Highspeed data transmission and wireless networking are the common facilities available on an intelligent building. With the development of demand for users, its function, structure and form are continuously in consummation. In this project we are going to design such a system by which we will provide early help from fire department.

Several prototypes of automated security surveillance systems built on various platforms have been proposed by different research groups all over the world. The smoke detectors signals are collected and processed in this system. Based on the signals from sensors, when the emergencies happen, a call will be made to the fire department so that they could extinguish the fire in time.

\section{Proposed System}

According to the design requirement, the block diagram of the system is shown in Figure I. The system in this designation is composed of smoke detector, call land circuit, 30 channel ISDN dialogic card, and central processing unit.

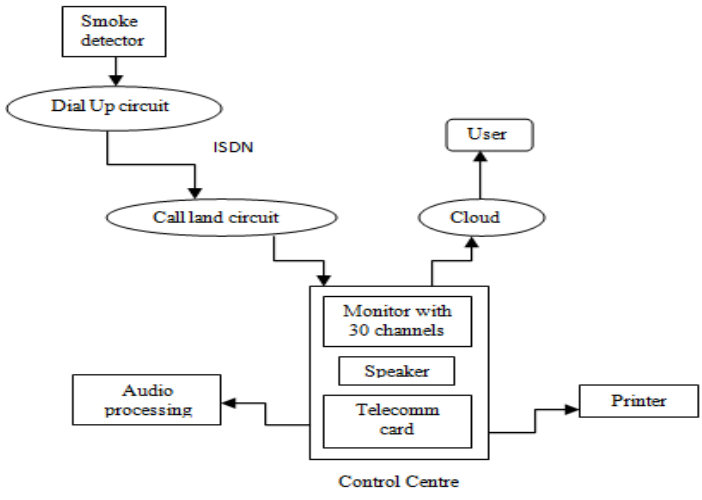

Fig I: Block Diagram of the System

Modular design is adopted throughout the system by which smoke detector s can be added and removed from the system without affecting the normal working. 
The Proposed system design is subdivided into two section: Hardware design and software design.

\section{Hardware Design of the system}

The hardware design of the system consists of smoke detector, call land circuit, 30 channel ISDN dialogic card. Of which smoke detectors are used for sensing the smoke and give signal to the GSM module. The dialogic card is used for the proper communication between fired place and the control centre.

\section{A. Smoke Detector}

First three types of detectors have been studied for three month for response time for different areas. From which we decided to use both type of smoke detectors as per the subscribers requirement. The working operation of both detectors is given below.

Extensive prior research suggests that no particle detector (photoelectric or ionization) is universally better at detecting all types of smoke particles since each sensor operates on a different principle and therefore may respond differently to various conditions. Ionization detectors are more sensitive to smaller particles of combustion $(<1000 \mathrm{~nm})$ and recommended for areas containing highly combustible materials such as flaming liquids, newspapers, and paint cleaning solutions, which create flaming fires. On the other hand, photoelectric detectors are more sensitive to large combustion particulate $(>1000 \mathrm{~nm})$ than ionization detectors and recommended for areas which contain furniture such as sofas, chairs, and mattresses etc. which burn slowly and create smouldering fires.

As Shown in Fig. II, common photoelectric smoke detectors consists of a near-infrared (IR) lightemitting diode (LED) as a light source that emits near-IR light having a wavelength of $850 \mathrm{~nm}$ or $940 \mathrm{~nm}$ and a silicon photodiode as a light receptor which responds very well to near IR wavelengths.

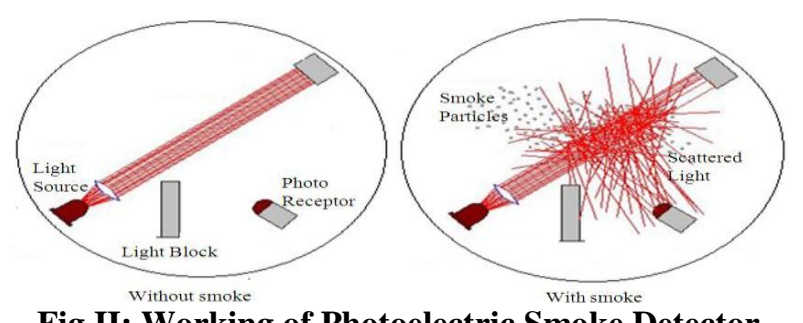

Fig II: Working of Photoelectric Smoke Detector

When no obstacle is present, the light from the light source on the left travels straight across and no light is detected by the photo sensor. When smoke enters the chamber, the smoke particles scatter the light and some amount of scattered light hits the sensor. When the amount of incident light reaches a predetermined threshold of the sensor, the sensor sets off an audible alarm in the smoke detector.

Ionization smoke alarms use ionic current as their detection mechanism. This ionic current is created in a chamber with a minute amount of a manmade element named Americum-241 and two electrodes.

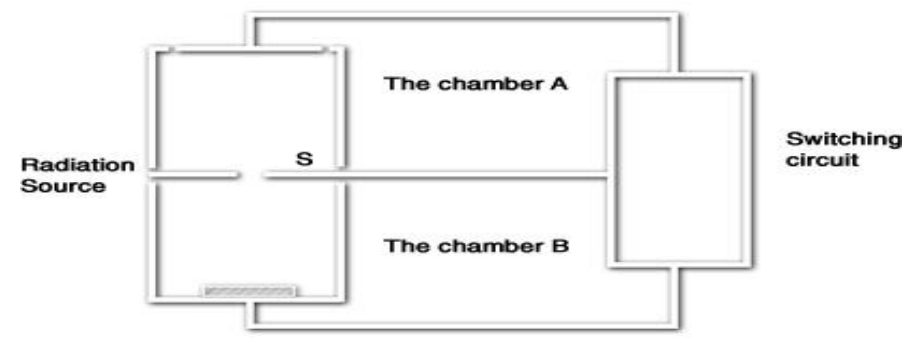

Fig III:-Working of Ionization smoke Detector

Alpha radiation from americium-241 foil increases the ability of air to conduct electricity and thus ionizes the air through chamber A to B allowing an electric current to flow. When electrical current flow in chamber A and B is constant, electrical potential of point $\mathrm{S}$ is kept balanced. When some particles enters the chamber alpha radiations are partly absorbed by the smoke particles and consequently flow of current in chamber drops and the electrical potential of point $\mathrm{S}$ is unbalanced. This detected by the detectors electronics circuitry to activate the alarm. When smoke enters the ionization chamber, the smoke particles attach to the ions and neutralize them, so they don't reach the plate. The drop in current between the plates triggers the alarms. Photoelectric alarms provided better response to smoldering fires than ionization type alarms. Ionization smoke alarms responded quicker to flaming fires compared to photoelectric smoke alarms. 


\section{B. GSM Module SIM 900}

There are many brands of GSM module, such as Siemens, Simcon, and so on. This design realizes with the application of SIM 900. This design realizes with the application of SIM 900. It is an industrial module that supports GSM/GPRS performance for voice, SMS, Data, and Fax in a small form factor and with low power consumption. It works on frequencies $850 \mathrm{MHz}, 900 \mathrm{MHz}, 1800 \mathrm{MHz}$ and $1900 \mathrm{MHz}$. It is very compact in size and easy to use as plug in GSM Modem. Its power supply is $4.2 \mathrm{~V}$ and $13 \mathrm{~V}$. Its current consumption is 40 $\mathrm{mA}$ and $590 \mathrm{~mA}$. Speech signals and data singles can be transmitted by SIM card reader and antenna. The SIM card voltage is $3 \mathrm{~V} / 1.8 \mathrm{~V}$. Data Interface of SIM 900 can transfer instructions and data bidirectional by AT Command. The Baud rate supported by the modem is between $9600 \mathrm{~b} / \mathrm{s}$ and $115200 \mathrm{~b} / \mathrm{s}$. It can support short message in text and PDU(Professional Development Units) modes, and can restart or make fault recovery by AT Command or turning off the signal.

\section{Telecommunication Card (D/300JCT-E1)}

Telecommunication card is a Single Span-JCT boards provide one span of digital network interfaces in a H.100-compliant universal PCI form factor. The boards contain rich media features such as voice processing, speech recognition software, fax, tone signaling, global tone detection, global tone generation, and call progress analysis, making them ideal for service providers and large enterprises.

The features of the telecommunication card are that it has high channel-per-slot density: one T-1 ISDN PRI trunk with 24 channels of voice processing or one E-1 ISDN PRI trunk with 30 channels of voice processing. It supports DSP-based onboard fax and host-based speech recognition to maximize the number of boards in the system. It offered in industry-standard 32-bit PCI form factor with universal connector. The ISDN Primary Rate Interface (PRI) firmware is a standard feature of the Single Span-JCT Series. The Single-Span JCT boards support Global Call software, a unified call control programming interface and protocol engine that makes it easier to provide worldwide application portability and can shorten development time by using the same application programming interface (API) for almost any network protocol.

The D/300JCT-E1 board processes the digital signaling information and digital voice signals from the telephone network.

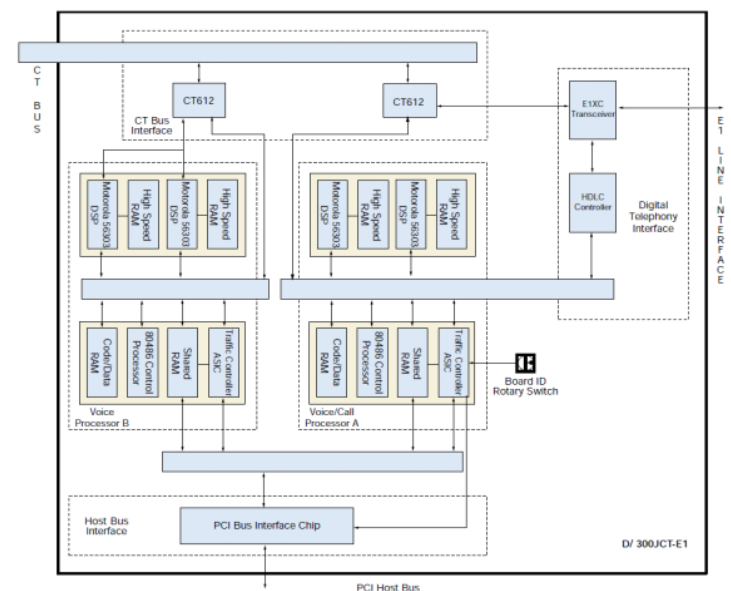

Fig IV: Functional Block Diagram

Digital E-1 signals enter the board via an E1XC line interface. Each of two CT612 functional modules on the D/300JCT-E1 board transmits several lower speed data streams over a single high-speed channel. The bus configuration is set when the firmware is downloaded at system initialization. The board's line interface extracts or inserts telephony signaling information, which is processed by an onboard control processor. The DSPs only process the digitized voice data. The HDLC receives ISDN signaling data from the E1XC interface and CT612 ASIC and makes it available to the control processor. It also formats and sends outbound signaling data from the control processor to the network interface through the CT612 ASIC and E1XC transceiver chip.

\section{Software Design of the system}

Cross platform technology is used to build the application. Cross platform is the Software that can run on multiple types of computer systems. For design of the software QT Framework with C++ with C11 standard is used. With the help of cross platform technology developers can save his time, manpower and cost to make the source code for different operating systems. 


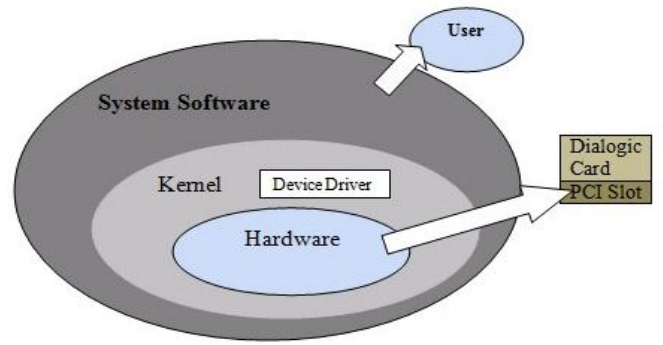

Fig V: Overview of software architecture

The system needs to detect the smoke density. Ones detected the dial up circuit will make call to call land circuit which is at fire department by SIM 900 module. The flowchart of main program for this system is shown in figure.

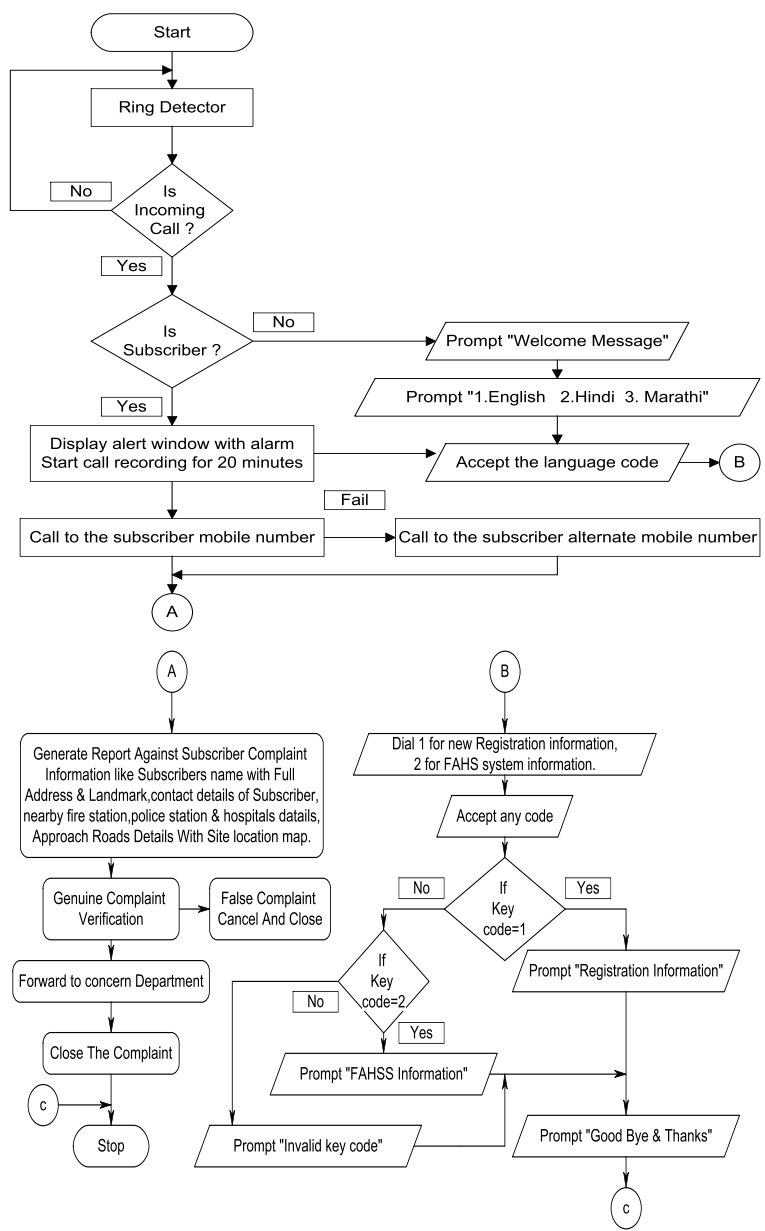

Fig VI:-Flowchart of the main program

\section{Working Principle}

The traditional fire alert system used mechanical devices or a human to monitor the surroundings means one can keep a watch on the building. If fire occurs, one can make a call to the fire department to give the information about the fired place. Then person seating in the office collect all information regarding the exact location of fired place. Then service will provided. This complete process will takes around half an hour, by that time most of valuable things will be destroyed and that is of no use. To overcome this problem, an idea to design and implement the automatic security system comes into picture, without disturbing the traditional system. In this system a call will be given to the control panel of the fire department so that they could extinguish a fire in time. In the proposed method we are going to design an automatic security alert system using GSM network, PCI board and cross platform technology.

In this method the smoke of fire get detected by smoke detector which gives signals to the dial up circuit. Here we are going to use GSM module as a dial up circuit to make a call to the particular number which 
is already stored into the call land circuit of control centre. At the control centre, we have main control unit, monitor with number of channels, printer and CPU for interfacing of control unit with server. In control unit we are going to use telecomm card which is useful in end to end communication of fired places with the control centre at a time. By making the use of integrated switched digital network line, one can make more than four calls at a time. So the work is done with ISDN line for the proper communication between fired place and control centre of fire department.

Once the call landed, fire alert system will get activated and generate all the necessary information regarding the fired place according to which the service will be provided to that place. In addition to this it make a call to the nearest one whose numbers are already stored in the system until the call will be received from one of them. The telecommunication card is inserted into the PCI slot of CPU which is connected through server that responds to requests across a computer network to provide, or help to provide network service.

\section{Advantages}

The advantage of the given system is that it overcomes the drawback of the all existing fire alert system. In the system a call will be given to the control panel instead of making SMS to the fire department so that they could extinguish a fire in time. PCI card will be used for the proper end-to-end communication. Cross platform is used as it is convenient to use single source code on multiple operating systems.

\section{Result}

The system executed successfully and it fulfills all the criteria for which it is designed. It can receive minimum one call and maximum 25 calls at a time. In the emergency situation, it make call to the nearest person whose number is already stored during the subscription. The system is also provided with voice recording facility which is useful for the forensic investigation.

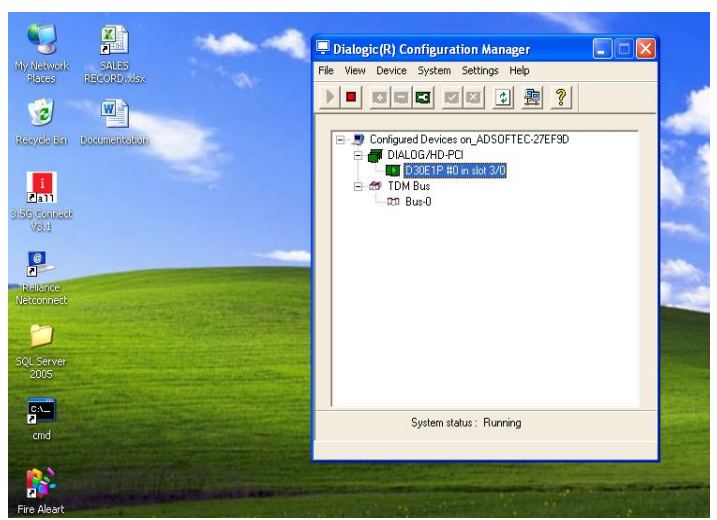

Fig VII: - User Authentication

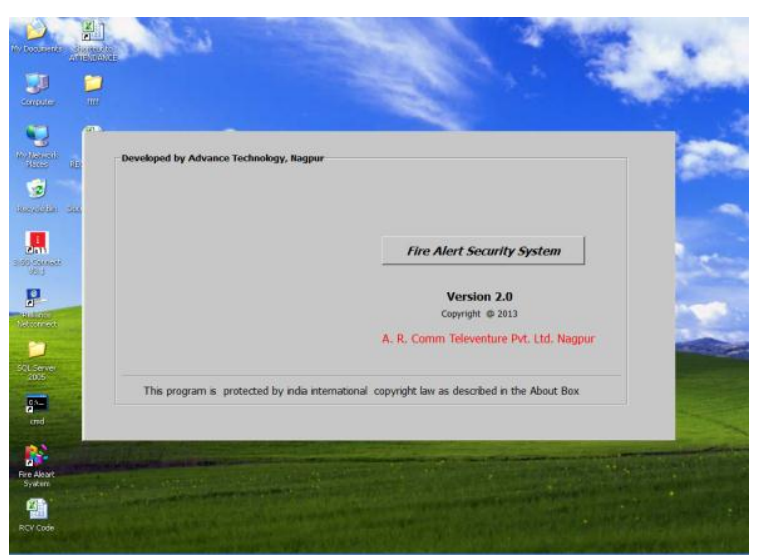

Fig VIII:-Application splash screen 


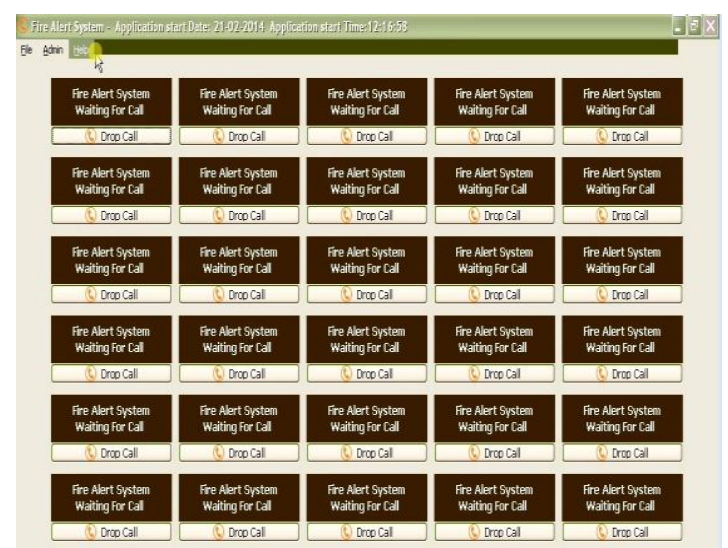

Fig IX:- GUI showing thirty channels

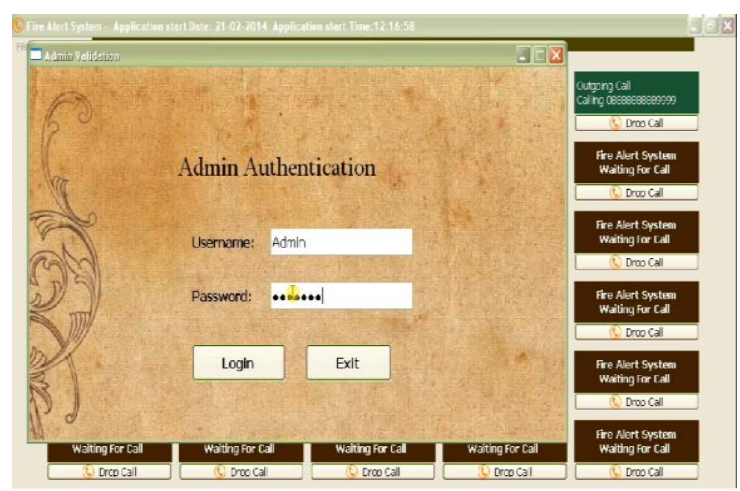

Fig X: System login page

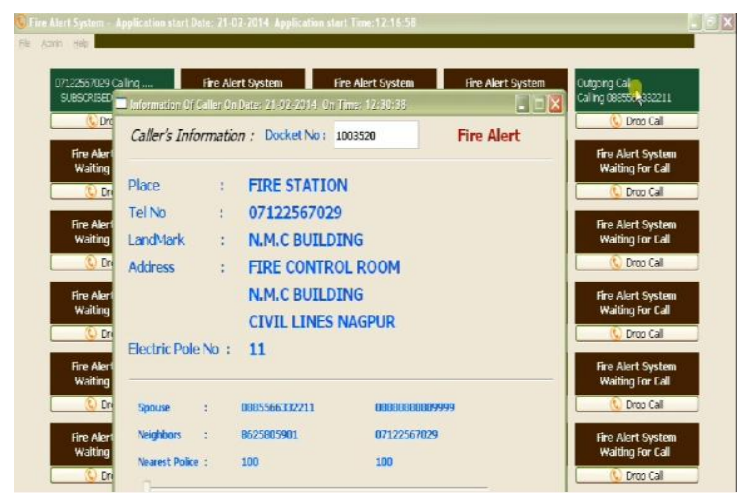

Fig XI: Pop up window showing callers information

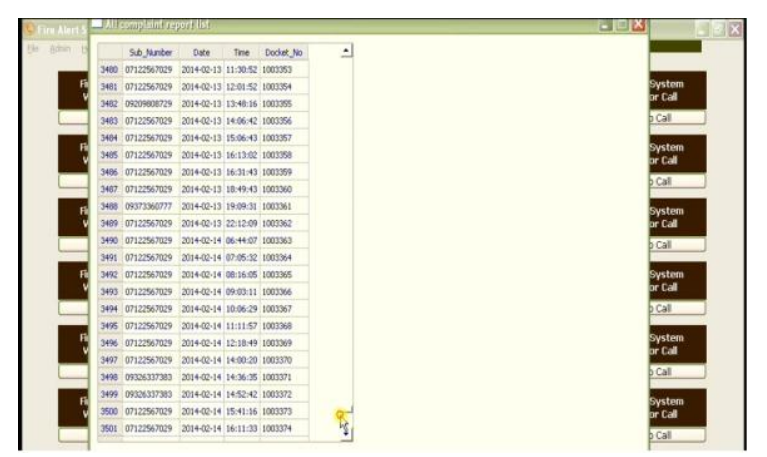

Fig XII: Database showing all complaints report list

Username and password are provided to the person who handling the software of system. Fig VIII shows the pop up window which will appears when an emergency occurred. Fig IX gives the screen shot of database of all report list which are recorded when call has came. 


\section{Conclusion}

The system is the design and implementation of Automatic Security Surveillance System Using PCI Board GSM Network and Cross Platform Technology. The traditional alert system is modified into automatic security alert system with call making if fire has occurred. The working model of the proposed security alert system is implemented in the corporation and is efficiently working in condition and it can be easily operated in all the available operating system.

\section{Future Scope}

The system designed is Automatic Security Surveillance System Using PCI Board GSM Network and Cross Platform Technology. And the system is implemented for detection of fire. Further development can be done by modifying it for the security purpose especially for the ladies as now a day's ladies are unsafe when they travelling alone. A small device can fits into wrist watch. When someone in trouble just press that button the call will automatically Given to the control centre according to which they will get help. Again with the concept of security alert system, one can make application useful in the robbery, for the senior citizens safety that is living alone.

\section{References:-}

[1]. Rakesh V S “An Improved Real-Time Surveillance System for Home Security System using Beagle Board SBC, Zigbee and FTP Webserver"Department of ECE Vidya Academy of Science and Technology Thrissur, Kerala, India-680501 Email: rakeshvs@ymail.com 2012 India Conference(INDICON),Annual IEEE pages:1240-1244.

[2]. Jun Hou, Chengdong Wu, Zhongjia Yuan, Jiyuan Tan, Qiaoqiao Wang, Yun Zhou. "Research of Intelligent Home Security Surveillance System Based on Zigbee", International Symposium on Intelligent Information Technology Application Workshops (IITAW), IEEE Computer Society, pp. 554-557, Dec. 2008

[3]. Vivek Nainwal, Pramod P.J, S.V Srikanth. "Design and Implementation of a remote surveillance and monitoring system using Wireless Sensor Networks", Electronics Computer Technology (ICECT), 3rd International Conference, pp. 186-189, April 2011.

[4]. LIAN Chun-yuan "Design of Intelligent Fire Alarm System Based on GSM Network" Changzhou Institute of Technology School of Electronic Information \& Electric Engineering Changzhou, China,2011 International Conference on Electronics and Optoelectronics (ICEOE 2011).

[5]. Fan Guan-lin "Design of Fire Alarm System Based on Multi-source Information Fusion" Department of Computer Science and Technology Nanyang Institute of Technology Nanyang, China,2010 3rd International Conference on Advanced Computer Theory and Engineering(ICACTE).

[6]. "Design and Implementation of Automatic Fire Alarm System based on Wireless Sensor Networks" Lei Zhang1, and Gaofeng Wang, 2009 International Symposium on Information Processing (ISIP’09) Huangshan, P. R. China, August 21-23, 2009, pp. 410413 .

[7]. www.fire.org.nz /Business-Fire Safety/Unwanted Alarms/ Documents /69efc74f88583 7062a01ae02b0e57a39.pdf.

[8]. www.aerospace.ceas.uc.edu/content/dam/aero/docs/fire/Papers/10Ssmokeresponse.pdf

[9]. www.rhydolabz.com SIM900 GSM/GPRS RS232 Modem - User Manual.

[10]. www.sim.com/wmDATASHEET SIM900 The GSM/GPRS Module for M2M applications.

[11]. Dialogic Inc1504 McCarthy Boulevard Milpitas, California 95035-7405USA www.dialogic.com, Copyright @ 2012 Dialogic Inc.

[12]. Vertical Communications, Inc. 1-877-VERTICAL www.vertical.com \#98-6610 Edition 8 January 2008.

[13]. Dialogic® JCT Media Boards Part Number 64-0015-02 Copyright @ 2000-2007.

[14]. Datasheet Intel® Dialogic $®$ Single Span-JCT Series Copyright @ 2002 Intel.

[15]. Abhilasha Walke, Prof. Aruna D. Tete "An Intelligent security system using PCI board, GSM Network and cross platform technology." International Journal of Computer Engineering and Applications, Volume V, Issue III, March 14 www.ijcea.com ISSN 2321-3469 